\section{EFFICIENT DETERMINATION} OF STABILITY LOBE DIAGRAMS DEPLOYING AN AUTOMATED, DATA-BASED ONLINE NC PROGRAM \section{ADAPTION \\ CHRISTIAN BRECHER, RALPH KLIMASCHKA, ALEXANDER STEINERT, STEPHAN NEUS}

Laboratory for Machine Tools and Production Engineering (WZL) of RWTH Aachen University, Germany

DOI: 10.17973/MMSJ.2021_10_2021052

r.klimaschka@wzl.rwth-aachen.de

Process instabilities due to regenerative chatter pose significant limitations on the achievable material removal rates and thus on the profitability of machining operations. Stability lobe diagrams serve to exploit the maximum yet stable cutting depth and can be determined either analytically or experimentally. While analytical approaches suffer from inaccuracies because of the assumptions made for the specific models, experimental stability lobe diagrams require extensive cutting tests. Therefore, this paper introduces a new automated experimental method for determining stability lobe diagrams in milling with reduced effort regarding time. A closed-loop system is designed, containing a sensor-based online chatter detection along with a strategy to set parameters for subsequent cuts based on the stability boundaries known at each iteration. Both cuts with continuously increasing cutting depth and varied spindle speed are deployed to ensure fast detection of stability limits. The method is tested for a slot milling use case and the results are compared to a conventionally obtained stability lobe diagram yielding a significantly reduction in required time $(-90 \%)$ and resources $(-67 \%)$ whilst maintaining good accuracy. The reduced effort qualifies the proposed method as a tool to rapidly deliver maximum productive yet stable cutting parameters for optimization of existing or enhanced planning of new manufacturing processes.

KEYWORDS

Stability lobe diagrams, regenerative chatter, online chatter detection, Poincaré section

\section{INTRODUCTION}

Machine tools operate in a highly competitive global environment urging manufacturing companies to maximize their productivity whilst providing high levels of product quality. A major obstacle in pushing conceivable limits of metal cutting processes is regenerative chatter. The prediction of this selfexcited vibration has been subject of extensive research for the past decades. Avoiding chatter is inevitable because it can result in poor surface finish, inaccuracies in workpiece dimensions, premature tool failure and damages to the main spindle [Kayhan 2009; Yusoff 2011]. Stability lobe diagrams (SLD) display the stability boundaries considering the parameters cutting depth $(a p)$ and spindle speed $(n)$ for a given combination of tool, workpiece, and machine structure. Thus, they can be utilized to design processes at spots of optimal material removal rate and stable conditions [Quintana 2011]. The challenges associated with existing methods for SLD determination are the reason why exploiting dynamic process limits with the help of SLDs plays a minor role in the industrial context. However, especially in customized small series production with small batch sizes and a wide range of changing tool-workpiece combinations, there is need to frequently identify optimal and reliable cutting parameters in order to maximize productivity. Non-stationary boundary conditions, e.g. caused by tool change, leads to a different dynamic system behavior yielding shifted stability boundaries. [Namazi 2007] Hence, SLDs must be determined within the shortest period of time possible using minimal amount of resources.

Analytic approaches for determining stability lobe diagrams aim to predict the onset of process instabilities without conducting trials of the actual machining process. However, since existing analytic models are based on the knowledge of the machine structure's direct and cross transfer functions as well as dynamic cutting force coefficients, extensive measurements are required first [Altintas 1995; Altintas 2008]. Moreover, assumptions and simplifications regarding the system's linearity and time-varying dynamic behavior often lead to inaccuracies of the simulated stability boundaries. On the other hand, experimental stability lobe diagrams represent the real chatter-critical boundaries and act as a reference for analytical models [Brecher 2018b]. The main drawback of conventional experimental determination are time-consuming cutting experiments. After each trial, the cutting depth is increased stepwise and then held constant for a fixed spindle speed until the onset of chatter is detected during the cut. Here, the number of required tests directly correlates with the desired SLD's resolution. [Brecher 2014]

In order to reduce the effort for experimental SLDs, Quintana et al. introduced a method with an inclined workpiece allowing the cutting depth to increase during a single cut continuously [Quintana 2008]. The concept is further analyzed in [Brecher 2014] and [Brecher 2018a] concluding that higher inclination angles lead to more significant deviations from conventionally obtained stability limits. Another approach for faster identification of stability boundaries is the continuous variation of spindle speed at constant feed per tooth as introduced in [Grossi 2015], calling it the spindle speed ramp-up test (SSR). Analogous to the inclination angle in the first approach, the angular acceleration should be kept to a minimum for better conformity with conventional stability boundaries [Brecher 2017]. A novel method combining both strategies is developed in [Brecher 2018b]. Starting in an assured stable region, the cutting depth is continuously increased at a fixed spindle speed until chatter vibrations are identified, triggering a feed stop. Subsequently, the current cutting depth is held constant while the spindle speed varies for a second cut initiating at the same tool position. This time, the return to stable conditions stops the cut. The procedure provides promising results identifying two stability boundaries within one groove. However, only tools that allow both face milling and plunge milling can be utilized. This is because of the tool being in full engagement whilst one machine axis is revolving between the two cuts to reset the inclination. Additionally, the machine setup is exposed to unstable process conditions for a significant time during the second cut. Consequently, this paper proposes a novel method for efficient determination of stability lobe diagrams minimizing the necessary time, material, and duration of instable machining. 
Expanding on the existing approaches of continuously varying cutting depth and spindle speed, an automatic and iterative procedure is established that returns an entire stability lobe based on one initial point of instability. For this, an auxiliary algorithm updates the workpiece condition after every iteration in order to prepare the grooves for subsequent cuts accordingly. In practice, the proposed method enables rapid and automated a priori determination of ideal cutting parameters for a given process to exploit the maximum achievable material removal rate and thus, increase productivity. Before the start of a new production series, for which the process limits are unknown, the proposed method can be included in a time- and resourceefficient automated program routine to generate maximum cutting depths for the actual production. The determined ideal cutting parameters can then be taken into account for enhanced process design.

\section{PROPOSED METHOD}

The proposed method for efficient stability lobe determination consists of five core elements integrated into a closed-loop system as illustrated in Figure 1. The core elements are (1) the machine tool, (2) a data acquisition (DAQ) board, (3) an external evaluation unit, (4) the machine's numerical control unit and (5) a dynamically parametrized NC program. Tested object is the combination of machine, workpiece and tool characterizing a specific manufacturing job within small series production. On the other hand, the data acquisition board as well as the evaluation unit represent the external testing equipment.

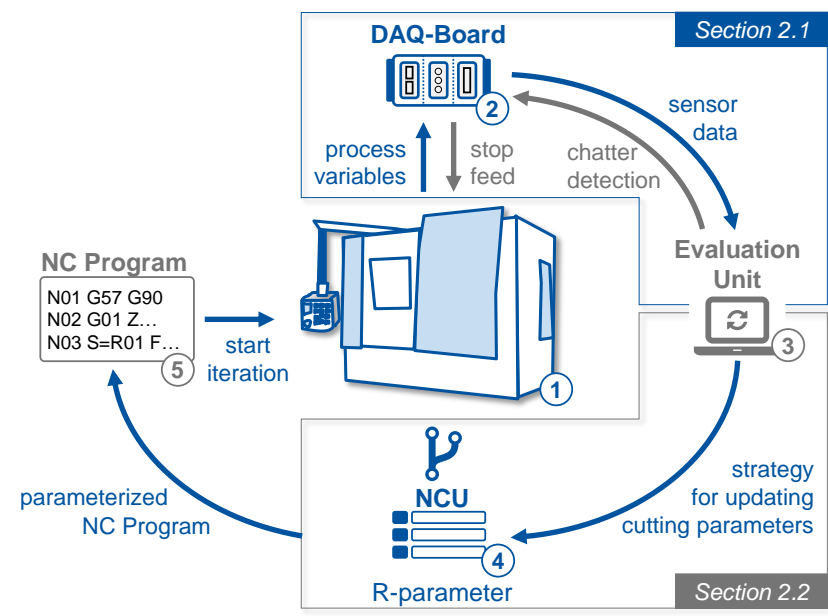

Figure 1. Principle of the proposed method

During each test cut as part of the efficient SLD determination, process data is gathered. Two signal input modules are embedded in the DAQ-board to monitor the spindle head movement measured by an acceleration sensor and the encoder signal tapped from the machine's control cabinet. Both signals serve as input data for a chatter detection algorithm run by the evaluation unit (see section 2.1). The algorithm is implemented in MATLAB environment. If the onset of regenerative chatter is detected, the feed is stopped immediately via a digital signal sent back to the machine control (digital I/O terminal X142 for Siemens NCU). For this, a digital output module is further included to the DAQ-board. The machine control's I/O terminal status is read by a synchronized action once every interpolation cycle of the Numerical Control Kernel (NCK) allowing for an immediate reaction. In addition to the feed stop, the chattercritical current cutting depth and spindle speed are recorded representing a point on the verge of the stability lobe diagram. To obtain the chatter-critical current cutting depth, precisely, the time between tool engagement into the workpiece and onset of chatter is measured. Knowing the cut's feed profile as well as start and end value of the cutting depth, the current critical depth of cut can be calculated. Subsequently, the tool retreats to a safe standby position awaiting the next cutting job. Based on all previously obtained stability boundaries, the cutting parameters of the next cut are derived automatically utilizing a parameter adaption strategy in the meantime (see section 2.2). Among others, these parameters include start and end values for the cutting depth, spindle speed, feed rate as well as $\mathrm{X}-, \mathrm{Y}$ - and $Z$-axis datum. The resulting cutting job information are then converted into R-parameters and transferred to the NCU via established machine communication protocol (TCP/IP). A parametrized NC program, referring to the current Rparameters, is executed to realize individual machining processes at each iteration as exemplarily shown by the synchronized action to manipulate the spindle speed override taken from the NC program:

N290 WHENEVER (\$AA_IW $[X]>=-R 36)$ AND (\$AA_IW $[X]<=-R 37]$ DO $\$ A A \_O V R[S 1]=100+((\boldsymbol{R} 21-\boldsymbol{R} 20) * 100 / \boldsymbol{R} 20) / A B S(\boldsymbol{R} 35-$ R32) *ABS(SAA_IW[X]+R32)

In this NC program excerpt, the spindle speed is linearly varied as a function of the current tool position \$AA_IW[X] and only applies for the subsequent block, i.e. machining the groove. Referencing the current R-parameters is essential because there is no information about the SLD's shape in the beginning and the procedure must dynamically react to the stability transitions found at every iteration. Since the workpiece groove status changes with every conducted cut, the processing history is tracked permanently in order to update geometrical offsets. For this purpose, a digital twin of the workpiece was implemented to store the geometrical status of every groove. Besides, the groove is prepared through a facing routine based on the digital workpiece twin before every cut to prevent cutting depth modulation caused by prevailing chatter marks. By changing the status of the digital I/O terminal, the current iteration is completed and a new iteration is finally initiated. The procedure is repeated until enough information about the desired stability lobe diagram are gathered. During the entire process, no manual intervention is required.

\subsection{Time domain chatter detection}

Existing sensor-based chatter detection for automated process stability investigations are realized either in frequency or time domain. Frequency domain approaches exploit the characteristic sudden increase in vibration amplitude of selfexcited vibrations after exceeding the critical depth of cut. Instability can be identified by a high amplitude at a frequency close to a natural frequency of the machine-tool-workpiececombination in the spectrum. For stable processes, the tooth passing frequency and its harmonics are dominant. Acoustic signals [Quintana 2008] and acceleration signals [Grossi 2015] both lead to similar results if utilized for the frequency order analysis. Despite the robustness of the frequency domain methods, their drawbacks are low frequency resolution for short measurement times and the fact that chatter can only be detected after the onset rather than during emergence of the instability.

In contrast, time domain methods allow online chatter evaluation without significant time delay and the knowledge of potential chatter frequencies. Both the experimental approaches presented in [Brecher 2014; Brecher 2015] and the time domain milling simulation proposed in [Honeycutt 2016] apply Poincaré maps and sections as introduced in [Davies 1998]. The idea is to analyze the repeatability of the Tool Center Point (TCP) position for each revolution. A chaotic TCP motion is an indicator for an instable process. A sharp, 
repeatable TCP motion indicates stable conditions. Thus, the trajectory of the TCP displacement induced by the cutting forces is plotted in a two-dimensional plane, as shown in Figure 2.

Furthermore, bisection points are defined at certain angular positions along the trajectory. The bisection points' position is tracked every spindle revolution as a measure to evaluate repeatability. Brecher et al. incorporated a chatter indicator (CI) based on the bisection points' standard deviation (sBx, sBy) normalized by the standard deviation of the entire trajectory (sTx, sTy), as defined in equation 1 [Brecher 2015].

$$
C I=\frac{s_{B x} \cdot s_{B y}}{s_{T x} \cdot s_{T y}}
$$

Criteria such as positive trend, positive surge and absolute limits serve as a means to assess the course of the chatter indicator regarding stability. The suggested criteria were further analyzed, empirically quantified, validated, and extended in [Brecher 2018b]. If the threshold for one of the criteria is exceeded during the process, the algorithm returns instability.

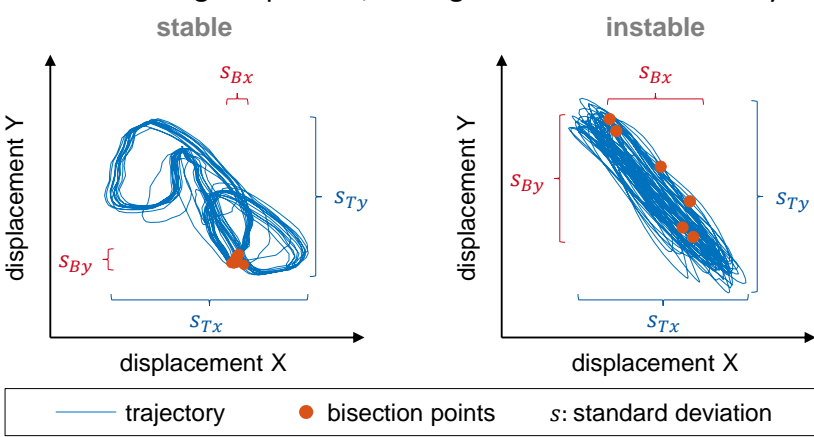

Figure 2. Distinction between stable and instable cutting conditions

For the closed-loop method proposed in this paper, the time domain chatter algorithm as described in [Brecher 2018b] is implemented and integrated into a graphical user interface on the external evaluation unit. For approximating the TCP displacement, an encapsulated, tri-axial IEPE accelerometer (PCB piezotronics) along with a NI-9234 (National Instruments) input module are utilized to measure the acceleration at the spindle head with a sampling rate of $5.12 \mathrm{kHz}$. Before double integration, the signal is high-pass filtered with a cutoff frequency of $50 \mathrm{~Hz}$, detrended and periodically regressed to create sharp trajectories and prevent induced interferences. The necessitated bisection points are derived from the tapped encoder signal, which is preprocessed by an interpolation unit and interpreted by a NI-9411 input module. For triggering the feed stop after chatter detection, an output module of type NI9472 is used. All three modules are embedded in a NI-cDAQ9178 compact DAQ-chassis representing the interface between the machining process and external evaluation unit, as shown in Figure 1 . The chatter indicator is calculated every $0.1 \mathrm{~s}$ based on the last $0.3 \mathrm{~s}$ data block and checked for the mentioned stability criteria in [Brecher 2018b]. Thus, a fast and reliable chatter detection algorithm is established as a core component of the proposed method.

\subsection{Strategy for cutting parameter adaption}

For the automated online NC program adaption, a strategy is required based upon which the cutting parameters are set for each cut. The objective is to rapidly gather enough information about the shape of the investigated stability lobe in order to provide regions of maximum material removal rate for machining processes. The proposed concept, as shown in Figure 3 , is divided into two phases. The first phase consists of cuts with continuously increased cutting depth. In the second phase, the spindle speed is varied during the cuts. Schematically, the cuts are illustrated by arrows in Figure 3. The root of an arrow indicates the start of a cut and the arrow tip stands for the planned finish of a cut. However, once the transition to instability is detected (represented by blue markers in Figure 3), the feed is stopped, the tool retreats to standby position and the next cut is calculated. Ideally, the planned end of a cut is not reached. The spindle speed variation promises more accurate results than a varied cutting depth [Brecher 2017; Brecher 2018a]. One reason is that the effective cutting depth of a tooth is not constant for one tool revolution due to the inclination. This leads to a distortion of the measured stability boundaries. However, the major advantage of cutting depth variation is that cuts can be designed to start under stable conditions without any knowledge about the stability lobe (i.e. $a p=0 \mathrm{~mm}$ ). Thus, the first phase is intended to give an estimate about the lobe's position and width. For this, cuts with increasing cutting depth at a fixed spindle speed are conducted until the lobe's vertex is found, as displayed in the left diagram of Figure 3. The initial point of instability (red marker in Figure 3) provides the spindle speed for the first cut with increasing cutting depth. Per convention, the second cut is always conducted with lower spindle speed than the first one.

In a realistic scenario, an executed cut can remain completely stable. In that case, the job is repeated with a greater starting depth of cut. The achievable increase in cutting depth during one cut is a consequence of the inclination angle and the length of the workpiece. By analyzing already detected critical cutting depths, the left and right flank of the stability lobe can be identified. By this, it is deduced whether the spindle speed must be increased or decreased for the remaining cutting jobs of phase 1. In Figure 3 (1), for example, the second cut yields a lower critical cutting depth than cut 1 . Hence, the lobe's right flank can be identified resulting in another reduction in spindle speed for the third cut.

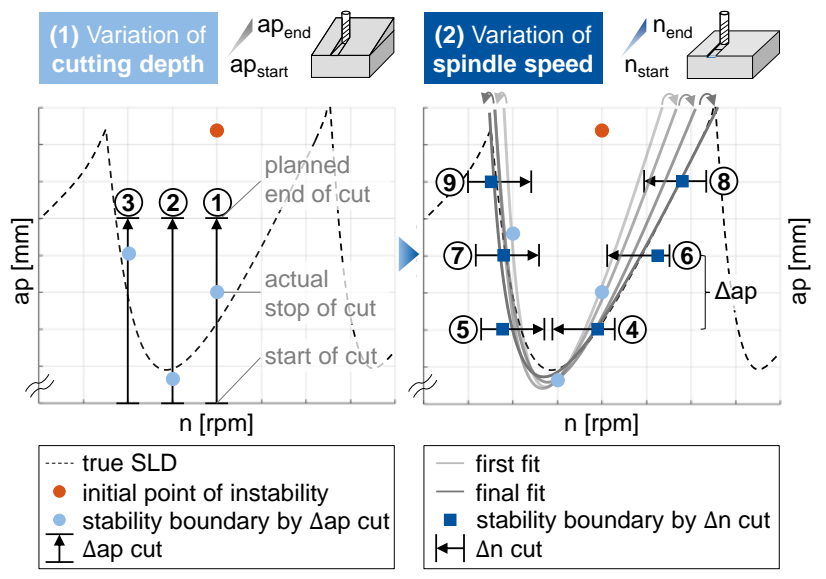

Figure 3. Strategy for automated online NC program adaption

In the second phase, the stability lobe is investigated in more detail. At first, a stability lobe based on the existing stability boundaries from phase 1 is fitted using an analytical expression for the critical depth of cut. Equation 2 represents the absolute chatter stability law originally formulated by [Tlusty 1957] and [Tobias 1958]. In case of groove milling, the chatter critical axial depth of cut is denoted by $b_{c r}$ and the machine system's transfer function by $G(j f)$. The constant $k_{c b}$ considers the impact of the workpiece material on the cutting force.

$$
b_{c r}=\frac{1}{2 k_{c b} \cdot\left|\operatorname{Re}\{G(j f)\}_{\text {neg }}\right|}
$$

Approximating $G(j f)$ with the dynamics of a 1-DOF spring-massdamper system yields the following expression. 


$$
\left.f(n)=\left(2 \cdot x_{1} \cdot \mid \operatorname{Re}\left\{\left(\frac{x_{2}}{\frac{i \cdot n^{2}}{x_{3}}+x_{4} \cdot \frac{i \cdot n}{x_{3}}+1}\right)\right\}\right\}_{n e g} \mid\right)^{-1}
$$

Unknown system characteristics like the responsible vibrational mode, the modal damping and $k_{c b}$ are summarized in the coefficients $\left(x_{1}, x_{2}, x_{3}, x_{4}\right)$ which are fitted in a numerical optimization step. The analytic function $f(n)$ returns the chattercritical depth of cut for a given spindle speed $n$ and exhibits the characteristic lobbed shape as illustrated in the second diagram of Figure 3. Cuts on alternating lobe sides are then conducted to check the fitted stability boundaries. Both the feed per tooth and the angular acceleration of the spindle are held constant for the trials. The lobe is updated after each detected stability boundary as the fit is expected to get more accurate with additional cuts. This is indicated by the fit moving towards the true SLD (dashed line) in Figure 3. Every two cutting jobs, the depth of cut is increased by $\Delta a p$ to concretize the regions of maximum productivity. The total time needed for the SLD determination depends on the choice of $\Delta a p$. High values lead to a shorter determination time but negatively affect the accuracy of the result concurrently.

The required spindle speed intervals (arrows in Figure $3(2)$ ) are located based on the fit. Precisely, for each level in cutting depth, the corresponding spindle speeds on left and right lobe flank are derived using equation 3 . It is anticipated that the transition to instability happens at $50 \%$ of the interval providing enough buffer for inaccuracies in the fit. Thus, the risk of starting in unstable regions is minimized. The procedure ends if the narrow region close to the lobe's apex is reached. This is indicated by cuts repeatedly starting under unstable conditions. However, as the spindle speed intervals could overlap adjacent stability lobes, cuts are re-conducted once beginning closer towards the stability boundary in that case. Hence, more information about the apex are conceivable resulting in enhanced knowledge of maximum material removal rates for the machining process.

\section{EXPERIMENTAL VALIDATION SETUP}

The proposed method is tested on a 4-axis horizontal machining center (Heller H2000) with box-in-box structure. A four-toothed, $50 \mathrm{~mm}$ diameter milling cutter head (Sandvik Coromant) of type R390-050Q22-18M is equipped with pre-used coated carbide inserts (R390-18 $0608 \mathrm{M}-\mathrm{PM} 4230$ ) and connected to the spindle via a HSK-A63 tool interface, as shown in Figure 4.

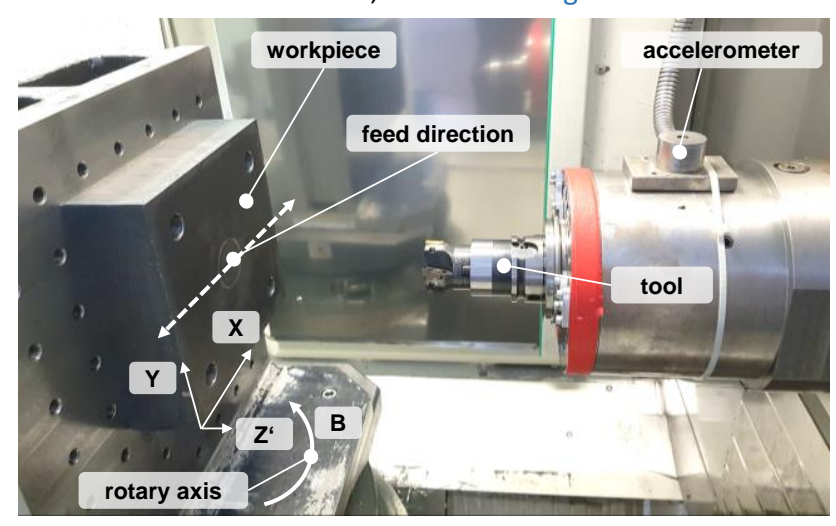

Figure 4. Experimental validation setup

The accelerometer needed for chatter detection (section 2.1) is mounted onto a slightly angled aluminum adapter which was adhered on top of the spindle head. As workpiece, a C45 (1.0503) steel block with the dimensions $220 \times 220 \times 60 \mathrm{~mm}$ is used. The block is fixed at a manufacturing tombstone with four M12 bolts. The tested system of machine tool, workpiece and tool represents an example for a possible machining setup in a customized small series production.

Cuts with inclined cutting depth are realized by revolving the Baxis. The inclination angle is kept at $1.5^{\circ}$. Consequently, the cutting depth can be altered by $5.75 \mathrm{~mm}$ within one phase 1 cut. Cuts with spindle speed ramp are enabled by synchronized actions for the spindle override. The feed stop after instability detection is realized by a delete distance-to-go (DELDTG) synchronized action. An implemented dwell time with rotating spindle using G04 command ensures enough time for the tool to cut free. According to the suggested insert specifications, the feed per tooth is set to $0.2 \mathrm{~mm} /$ tooth. For the tested use case, an initial point of instability at $1,300 \mathrm{rpm}$ and $9.5 \mathrm{~mm}$ is assumed giving a cutting velocity of approximately $200 \mathrm{~m} / \mathrm{min}$. Since this value also matches the manufacturer's specifications, a relevant SLD region is investigated. The radial depth of cut was kept at $50 \mathrm{~mm}(100 \%)$. In addition, an assured stable cutting depth of $3.5 \mathrm{~mm}$ is given.

\section{RESULTS AND DISCUSSION}

The proposed procedure is employed to determine one stability lobe. The results for the investigated use case are shown in Figure 5. Proceeding from the initial point of instability $(1,300 \mathrm{rpm} ; 9.5 \mathrm{~mm})$, five cuts with continuously increased cutting depth are conducted in phase 1 until the vertex is localized between 1,325 rpm and 1,375 rpm. In phase 2, six cuts with varied spindle speed are made resulting in a final fit illustrated as a blue curve. It is observed that stability transitions of the second phase (blue squares) show good accordance with the characteristic lobbed trend of a SLD. In contrast, the stability boundaries resulting from cuts with varied cutting depth (blue circles) scatter around the final fit by up to $0.5 \mathrm{~mm}$. These results support the developed strategy to use cutting depth variation for a first perception of the stability lobe and concretize by spindle speed variation. In order to compare the new method's result with the conventional procedure, a conventional SLD was added to Figure 5.

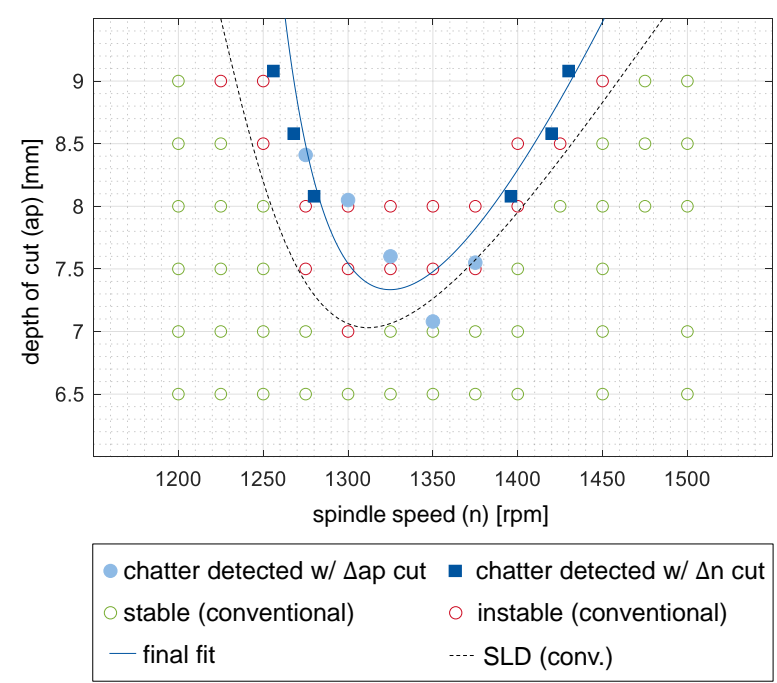

Figure 5. Result of the proposed method compared to conventional SLD The SLD had been determined as part of previous works and was published in [Brecher 2018b]. The SLD was conventionally obtained under almost the same conditions as described in section 3. The only significant difference is that in [Brecher 2018b] cutting inserts at an earlier state of wear were used. Both curves closely resemble shape. However, the conventional SLD exhibits lower stability boundaries than the final fit of the proposed method. As described for turning 

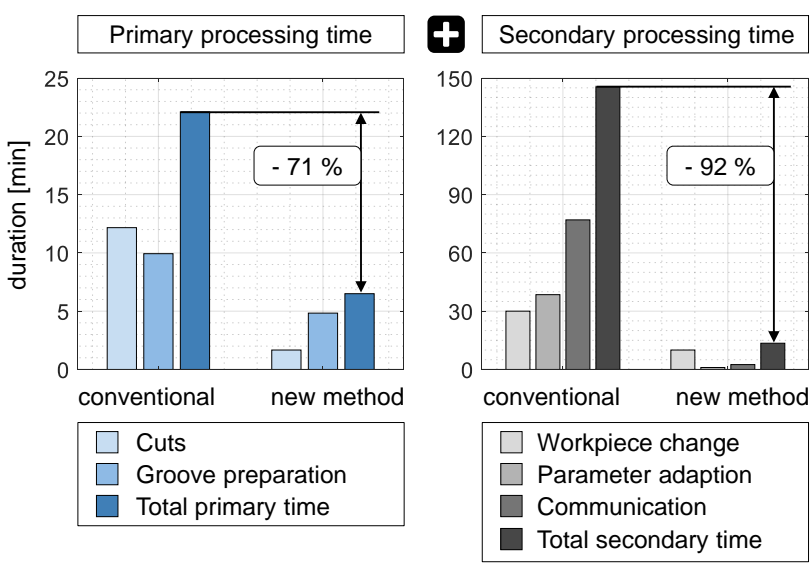

Figure 6. Reduction in time for SLD determination by proposed method operations in [Chiou 1995], [Taylor 2011] and [Clancy 2002], progressed tool wear leads to higher process damping ultimately shifting up stability boundary. Thus, the proposed method yields reasonable conformity with the conventionally obtained SLD. Nevertheless, the results indicate that stability lobe diagrams are influenced by numerous impacts. To tackle this challenge, focus of future work will be a sensitivity analysis of factors like tool wear and position-dependent machine dynamics. The goal is to supplement stability lobes with an envelope based on confidence intervals. Thus, an average SLD for a given process can be determined indicating statistically assured stable and instable regions. A primary benefit is that resulting SLDs could be utilized for stability assessment independent of specific process conditions. The necessitated time effort for the proposed method and the conventional approach is contrasted in bar charts shown in Figure 6 . The total processing time is divided into productive primary processing time and auxiliary secondary processing time. For the conventional method, a SLD resolution of $25 \mathrm{rpm}$ and $0.5 \mathrm{~mm}$ was assumed. As illustrated in Figure 5 , 59 measurement cuts were required taking $12: 10 \mathrm{~min}$. It is noted that starting at $6.5 \mathrm{~mm}$ cutting depth for the conventional SLD represents a rather radical reference. On the contrary, the new method took 6:30 min in total which means a reduction of $71 \%$. 1:40 min was consumed for the measurement cuts and 4:50 min for facing.

Considering the material needed, the conventional method suffers from the large number of cutting trials at high cutting depth. In total, 2.63 liters of material volume were machined. Since 1.1 liters were available from one workpiece, the workpiece had to be changed twice. The new method required 0.87 liters of material volume realizing the obtained stability lobe in one workpiece. Being part of the secondary processing time, the time for workpiece change was reduced by $20 \mathrm{~min}$ assuming $10 \mathrm{~min}$ per change. Per cut, the time for choosing new cutting parameters was estimated to be $30 \mathrm{~s}$ for the conventional and $2 \mathrm{~s}$ for the proposed method. For parameter transfer into the NC program and tool approach, $60 \mathrm{~s}$ and $5 \mathrm{~s}$ were averaged, respectively. With a total number of 77 cuts, the conventional approach took $2 \mathrm{~h}$ and $25 \mathrm{~min}$ whereas only 30 cuts and thus $8 \%$ of that time $(13: 50 \mathrm{~min})$ were required for the automated NC program adaption. All in all, the total processing time of $2 \mathrm{~h}$ and $48 \mathrm{~min}$ could be reduced to $20 \mathrm{~min}$ representing $2.5 \mathrm{~h}$ of saved time. For the conventional approach, it was assumed that chatter evaluation was based on the identification of chatter marks and extensive noise generation.

Therefore, instable cuts had to be performed using the entire workpiece length leading to a significant time under instable conditions. For the new method, however, the process was stopped immediately after the onset of chatter. Here, a

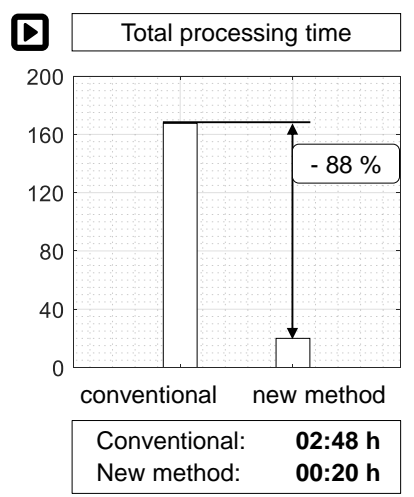

maximum time delay adds up to $0.3 \mathrm{~s}$. Consequently, the instable machining time could be reduced by $98 \%$ from $3: 43 \mathrm{~min}$ (18 instable cuts) to $3.3 \mathrm{~s}$ (11 instable cuts).

\section{CONCLUSION}

This paper presents a novel experimental method for efficient determination of stability lobe diagrams. The proposed method offers rapid and automated a priori determination of ideal cutting parameters in order to maximize productivity of a given machining process. An automated NC program adaption is realized as a closed-loop system containing a sensor-based online chatter detection and a strategy for updating cutting parameters. In an iterative process, stability boundaries are detected during cuts with continuously varied cutting depth for a first perception of the stability lobe and cuts with varied spindle speed for a more detailed investigation. An analytic chatter lobe is fitted based on the collected points of the stability verge. From this, parameters for subsequent cutting jobs are deduced. The parameters are transferred to the NCU via an established communication between an external evaluation unit and the machine, modifying a parametrized NC program. A practical use case proves the new method's significantly reduced effort regarding time (-90\%) and material (-67\%) compared to conventional experimental approaches. Furthermore, the instable machining time is minimized. Yet, a reasonable conformity with conventionally obtained references can be demonstrated. Applying the proposed method, the investigated stability lobe is found in $20 \mathrm{~min}$ rather than in $2 \mathrm{~h}$ and $48 \mathrm{~min}$ when using conventional methods for SLD determination. In practice, this means that in case of changing boundary conditions especially for small series productions, i.e. different tools or workpiece material, manufacturing companies are enabled to determine and exploit cutting parameters that maximize productivity with acceptable effort without need for manual interaction.

\section{ACKNOWLEDGEMENT}

The authors wish to gratefully acknowledge the support of the German Research Foundation (Deutsche Forschungsgemeinschaft, DFG). This work was funded as part of the DFG Project "Efficient determination of Stability Lobe Diagrams". (Project Number BR 2905/73-1)

\section{REFERENCES}

[Altintas 1995] Altintas, Y.; Budak, E. Analytical Prediction of Stability Lobes in Milling. CIRP Annals, 1995, Vol.44, No.1, pp 357-362 
[Altintas 2008] Altintas, Y.; Eynian, M.; Onozuka, H. Identification of dynamic cutting force coefficients and chatter stability with process damping. CIRP Annals, 2008, Vol.57, No.1, pp 371-374

[Brecher 2014] Brecher, C.; Epple, A.; Fey, M. Efficient determination of process parameters (in German). wt Werkstatttechnik online, 2014, Vol.104, No.5, pp 260-265

[Brecher 2015] Brecher, C.; Daniels, M.; Wellmann, F.; Neus, S.; Epple, A. Realization of efficient machining processes: Final report of the BMBF joint project ReffiZ (in German). Aachen: Shaker, 2015. ISBN 9783-8440-3917-7

[Brecher 2017] Brecher, C.; Chavan, P.; Epple, A. Efficient determination of stability limits (in German). wt Werkstattstechnik Online, 2017, Vol.107, No.5, pp 313-317

[Brecher 2018a] Brecher, C.; Chavan, P.; Epple, A. Investigations on the limitations of rapid experimental determination of stability boundaries during milling. Mechanics \& Industry, 2018, Vol.18, No.6

[Brecher 2018b] Brecher, C.; Chavan, P.; Epple, A. Efficient determination of stability lobe diagrams by inprocess varying of spindle speed and cutting depth. Advances in Manufacturing, 2018, Vol.6, No.3, pp 272-279

[Chiou 1995] Chiou, Y.; Chung, E.; Liang, S. Analysis of tool wear effect on chatter stability in turning. International Journal of Mechanical Sciences, 1995, Vol.37, No. 4. pp 391-404

[Clancy 2002] Clancy, B.; Shin, Y. A comprehensive chatter prediction model for face turning operation including tool wear effect. International Journal of Machine Tools and Manufacture, 2002, Vol.42, No.9, pp 1035-1044

[Davies 1998] Davies, M.; Dutterer, B.; Pratt, J.; Schaut, A.; Bryan, J. On the Dynamics of High-Speed Milling with Long, Slender Endmills. CIRP Annals, 1998, Vol.47, No.1, pp 55-60

[Grossi 2015] Grossi, N.; Scippa, A.; Sallese, L.; Sato, R.; Campatelli, G. Spindle speed ramp-up test: A novel experimental approach for chatter stability detection. International Journal of Machine Tools and Manufacture, 2015, Vol.89, pp 221-230

[Honeycutt 2016] Honeycutt, A.; Schmitz, T. A New Metric for Automated Stability Identification in Time Domain Milling Simulation. Journal of Manufacturing Science and Engineering, 2016, Vol.138, No.7

[Kayhan 2009] Kayhan, M.; Budak, E. An experimental investigation of chatter effects on tool life. Proceedings of the Institution of Mechanical Engineers, Part B: Journal of Engineering Manufacture, 2009, Vol.224, No.11, pp 1455-1463

[Namazi 2007] Namazi, M.; Altintas, Y.; Abe, T.; Rajapakse, N. Modeling and identification of tool holder-spindle interface dynamics. International Journal of Machine Tools and Manufacture, 2007, Vol.47, No.9, pp 13331341

[Quintana 2008] Quintana, G.; Ciurana, J.; Teixidor, D. A new experimental metho-dology for identification of stability lobes diagram in milling operations. International Journal of Machine Tools and Manufacture, 2008, Vol.48, No.15, pp 1637-1645

[Quintana 2011] Quintana, G.; Ciurana, J. Chatter in machining processes: A review. International Journal of Machine Tools and Manufacture, 2011, Vol.51, No.5, pp 363-376

[Taylor2011] Taylor, C. M.; Sims, N. D.; Turner, S. Process Damping and Cutting Tool Geometry in Machining. IOP Conference Series: Materials Science and Engineering, 2011, Vol.26, p 12009

[Tlusty 1957] Tlusty, J.; Polacek, M. Examples of handlung selfexcited vibrations of machine tools (in German). München: Hanser Verlag, 1957.

[Tobias 1958] Tobias, S.; Fishwick, W. Theory of Regenerative Machine Tool Chatter. The Engineer, 1958, No.205

[Yusoff 2011] Yusoff, A.; Sims, N. Optimisation of variable helix tool geometry for regenerative chatter mitigation. International Journal of Machine Tools and Manufacture, 2011, Vol.51, No.2, pp 133-141

\section{CONTACTS}

Ralph Klimaschka, M.Sc.

Laboratory for Machine Tools and Production Engineering (WZL) of RWTH Aachen University

Campus-Boulevard 30, 52074 Aachen, Germany

+49 24180 27930, r.klimaschka@wzl.rwth-aachen.de, https://www.wzl.rwth-aachen.de 